$1994-06-20$

\title{
Inversion of Light Scattering Measurements for Particle Size and Optical Constants: Experimental Study
}

\author{
Matthew R. Jones \\ Brigham Young University - Provo, mrjones@byu.edu \\ Keng $\mathrm{H}$. Leong \\ M. Quinn Brewster \\ Bill P. Curry
}

Follow this and additional works at: https://scholarsarchive.byu.edu/facpub

Part of the Mechanical Engineering Commons

\section{Original Publication Citation}

Matthew R. Jones, Keng H. Leong, M. Quinn Brewster, and Bill P. Currry. "Inversion of Light Scattering Measurements for Particle Size and Optical Constants: Experimental Study." Applied Optics, 33(18), 20 June 1994. 4035-4041.

\section{BYU ScholarsArchive Citation}

Jones, Matthew R.; Leong, Keng H.; Brewster, M. Quinn; and Curry, Bill P., "Inversion of Light Scattering Measurements for Particle Size and Optical Constants: Experimental Study" (1994). Faculty Publications. 1345.

https://scholarsarchive.byu.edu/facpub/1345

This Peer-Reviewed Article is brought to you for free and open access by BYU ScholarsArchive. It has been accepted for inclusion in Faculty Publications by an authorized administrator of BYU ScholarsArchive. For more information, please contact ellen_amatangelo@byu.edu. 


\title{
Inversion of light-scattering measurements for particle size and optical constants: experimental study
}

\author{
Matthew R. Jones, Keng H. Leong, M. Quinn Brewster, and Bill P. Curry
}

\begin{abstract}
Measurements of the light scattered by a sample contain information regarding the physical properties of the sample. Laser light-scattering measurements can be made unobtrusively in environments that are inaccessible to other types of measurements, so laser light-scattering techniques have become an important tool in aerosol research. The primary difficulty associated with using light-scattering techniques to determine aerosol particle properties is inverting the measurements or extracting the desired information from the measurements. In this study we use a 15-channel polar nephelometer to measure the light-scattering patterns of monodisperse polystyrene spheres. The light-scattering measurements are inverted, and the particle size distribution function and optical properties of the particles are retrieved. The results of these inversions are presented after a brief description of the calibration and operation of the nephelometer.
\end{abstract}

Key words: Optical particle sizing, inverse problems, light-scattering measurements.

\section{Introduction}

Measurements of the light scattered by a particle or a collection of particles contain information regarding the physical properties of the particles. Lightscattering measurements can be made unobtrusively, so the development of experimental and computational methods for determining properties such as the particle size distribution function (PSDF) and optical properties from light scattering patterns is of considerable interest. The primary difficulty in using lightscattering techniques to determine aerosol properties is inverting the measurements or extracting the desired information from the data. This paper presents the experimental portion of a study that developed a technique capable of retrieving the PSDF and information regarding the optical properties of aerosol particles from light-scattering measurements. Symbols used in this paper are defined in Appendix A.

We use a 15-channel polar nephelometer to measure light-scattering patterns. The nephelometer's

M. R. Jones, K. H. Leong, and B. P. Curry are with the Argonne National Laboratory, 9700 South Cass Avenue, Argonne, Illinois 60439; M. Quinn Brewster and M. R. Jones are with the Department of Mechanical and Industrial Engineering, University of Illinois at Urbana-Champaign, 140 Mechanical Engineering Building, 1206 West Green Street, Urbana, Illinois 61801.

Received 21 May 1993; revised manuscript received 8 November 1993.

0003-6935/94/184035-07\$06.00/0.

CC 1994 Optical Society of America. detectors are located between $23^{\circ}$ and $129^{\circ}$ and are positioned such that they are equidistant in $\sin (\theta / 2)$. The light source is an unpolarized laser beam emitted by a GaAlAs laser diode with a wavelength of 0.84 $\mu \mathrm{m}$. Figure 1 shows the primary components of the nephelometer. Aerosol particles are entrained in the air stream that passes through the center of the scattering chamber and intersects the laser beam. The laser light scattered within the acceptance cone of each detector is measured by photodiodes, and the output from the photodiodes is digitized and stored on a personal computer. A more complete description of the nephelometer is given elsewhere. ${ }^{1}$

In the theoretical portion of this study, an inversion technique specifically designed to invert measurements obtained with the 15-channel nephelometer was successfully applied to simulated data sets. ${ }^{2}$ The inversion technique retrieves the PSDF and information regarding the optical properties of aerosol particles by expanding the distribution function as a linear combination of a set of orthonormal basis functions, and it uses the orthogonality properties of the basis functions to solve for the expansion coefficients. The inversion scheme requires absolute scattering measurements as inputs, so a technique to calibrate the nephelometer for absolute scattering measurements has been developed and is outlined in this paper. A complete description of the operation and calibration of the nephelometer is given elsewhere. ${ }^{1,2}$ The inversion technique is applied to measurements of the light scattered by polystyrene spheres of known size and optical properties. The 


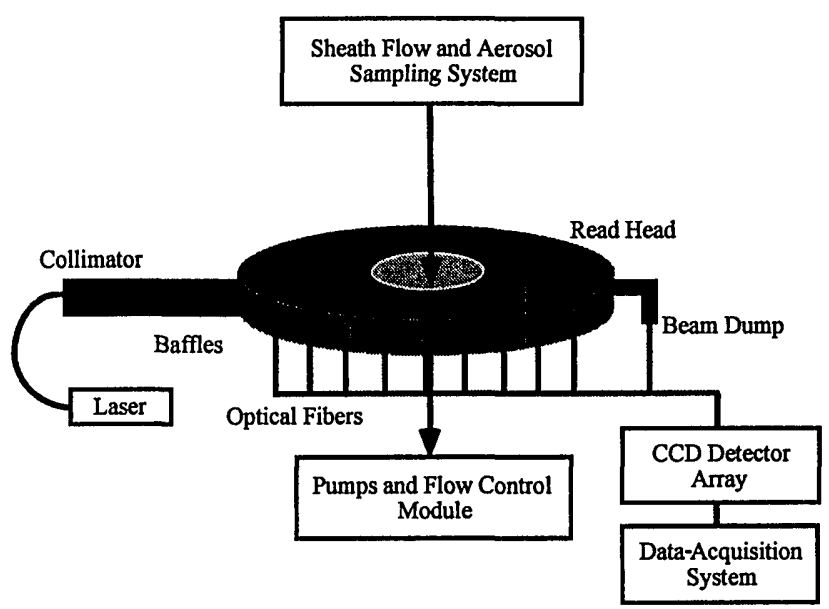

Fig. 1. Schematic diagram for a multichannel nephelometer.

results presented here demonstrate that the inversion technique developed in the theoretical portion of this study is a useful tool in analyzing measured scattering patterns.

\section{Calibration for Absolute Scattering Measurements}

Absolute scattering measurements require that the performance of the light source and all the optical components and detectors be well characterized. One method of characterizing a nephelometer is to measure the light scattered by spheres of known size, refractive index, and concentration. Although aerosol streams containing spherical particles of known size and refractive index can be generated through the use of commercially available polystyrene spheres, it is difficult to determine the particle number concentration accurately enough that this method can be used in a calibration procedure. However, the molecular concentration of a gas can easily be calculated at a given temperature and pressure, so the use of a gas with a relatively high refractive index for a scattering medium is a promising alternative. In the experiments described here, dichlorodifluoromethane $\left(\mathrm{CCl}_{2} \mathrm{~F}_{2}\right)$, commonly known as Freon-12, was used as the scattering medium for the calibration procedure.

Calibration for absolute scattering measurements requires that theoretical values of the ratio of the power received by each detector to the incident irradiance be known. The ratio of the power received by a detector to the incident irradiance is defined as an angular scattering cross section. Assuming single scattering, we find that the angular scattering cross section measured by the $j$ th detector $C_{j}$ is given by

$$
\begin{aligned}
C_{j}= & \int_{V} \int_{\Delta \Omega_{j}} \int_{n_{i}}^{n_{f}} \int_{k_{i}}^{k_{f}} \int_{n_{i}}^{n_{f}} \int_{x_{i}}^{x_{f}} N_{c} \tilde{f}(x, n, k) \\
& \times \frac{\mathrm{d} \tilde{C}}{\mathrm{~d} \Omega}(\Omega, x, n, k) \mathrm{d} x \mathrm{~d} n \mathrm{~d} k \mathrm{~d} \Omega \mathrm{d} V .
\end{aligned}
$$

When the following conditions are satisfied, Eq. (1) can be greatly simplified. First, the particle number concentration is uniform over the scattering volume. Second, the distribution of particle sizes and optical properties can be replaced by a monodispersion with an average size and optical properties:

$$
\tilde{f}(x, n, k)=\delta\left(x-x_{\text {avg }}\right) \delta\left(n-n_{\text {avg }}\right) \delta\left(k-k_{\text {avg }}\right) .
$$

Third, the differential scattering cross sections can be replaced by their average over the solid angle subtended by each detector:

$$
\begin{aligned}
\int_{\Delta \Omega_{j}} \frac{\mathrm{d} \tilde{C}_{j}}{\mathrm{~d} \Omega}\left(\Omega, x_{\text {avg }}, n_{\text {avg }},\right. & \left.k_{\text {avg }}\right) \mathrm{d} \Omega \\
= & \frac{\mathrm{d} \tilde{C}_{j}^{\text {avg }}}{\mathrm{d} \Omega}\left(x_{\text {avg }}, n_{\text {avg }}, k_{\text {avg }}\right) \Delta \Omega_{j} .
\end{aligned}
$$

The third assumption requires clarification. In practice, the integral shown in Eq. (3) is calculated by using the trapezoidal rule, so the integral is replaced by a sum. Therefore, Eq. (3) implies that the average of a product is equal to the product of the averages. This is only true if one of the terms in the product is a constant. Equation (3) will only be valid if the solid angles subtended by the detectors are sufficiently small that the differential scattering cross sections are approximately constant over the subtended solid angles. This approximation will be valid near $90^{\circ}$ where the slope of the scattering pattern is small, but it may be suspect in the forwardscattering angles where the scattering pattern varies rapidly with angle. The validity of assuming that the differential scattering cross sections were constant over the solid angles subtended by the nephelometer's detectors was thoroughly investigated, ${ }^{1}$ and it was found that the errors were less than $1.5 \%$ if the scattering pattern did not vary with $\theta$ more rapidly than $\theta^{-3}$.

These three assumptions eliminate the integrals, and Eq. (1) simplifies to

$$
C_{j}=N_{c} V_{j} \frac{\mathrm{d} C_{j}^{\mathrm{avg}}}{\mathrm{d} \Omega}\left(x_{\mathrm{avg}}, n_{\mathrm{avg}}, k_{\mathrm{avg}}\right) \Delta \Omega_{j} .
$$

Equation (4) is the fundamental equation for the nephelometer. When calibrating the nephelometer with Freon-12, one can calculate all the parameters on the right-hand side of Eq. (4). The particle number concentration is obtained from the ideal gas law. The scattering volumes and the solid angles subtended by the detectors are determined from an analysis of the nephelometer's geometry. ${ }^{1}$ The following paragraphs describe the calculation of the average differential scattering cross sections.

Differential scattering cross sections can be calculated from Mie theory ${ }^{3}$ only if the scattering particles are spherical. However, when Rayleigh scattering prevails, as in the case of the Freon calibration, the scattering pattern is fairly insensitive to the particle shape and depends mainly on the particle volume. The weak dependence of the scattered light on the particle shape is accounted for with shape-depolariza tion factors. Therefore, one can calculate differen- 
tial scattering cross sections for Freon-12 molecules from Mie theory with an effective scattering diameter. Although much simpler and faster approximations to Mie theory can be used in this case, Mie theory was used so the nephelometer's response to scattering by larger spheres could be calculated with the same code. The depolarization factor and the refractive index of Freon-12 have been measured at several wavelengths, ${ }^{5}$ and one can use these measurements to determine an effective diameter and refractive index at the wavelength of interest. The determination of these effective properties is only briefly described here, but a complete development is given by Jones. ${ }^{1}$

The qualitative features of the spectral dependence of the refractive index can be described by the classical damped oscillator or Lorentz model.5,6 The key assumptions of the Lorentz model are that the medium consists of charged particles that are held in equilibrium by perfectly elastic or Hooke's law forces and are subject to isotropic, linear damping forces. At frequencies far from resonant frequencies, the Lorentz model gives the following relationship between the refractive index and the wavelength of the incident beam:

$$
\frac{n^{2}+2}{n^{2}-1}=c_{0}+\frac{c_{1}}{\lambda^{2}}
$$

The constants appearing in Eq. (5) are determined from the least-squares fit of measured values of the refractive index of Freon-12. ${ }^{4}$ The values of the refractive index shown in Fig. 2 were measured at $0{ }^{\circ} \mathrm{C}$ and $1 \mathrm{~atm}$, but one can obtain the refractive index at different temperatures and pressures by using the Gladstone-Dale equation. ${ }^{6}$

Once the refractive index is known, an effective scattering diameter for the Freon-12 molecules can be determined. We use the Rayleigh-Gans scattering theory to obtain a relationship between the refractive index and the effective scattering diameter. ${ }^{1}$ This relationship also depends on the particle number concentration and the depolarization factor $\rho$. For Freon-12, $\rho$ is $0.048 .^{4}$ It should be noted that the effective scattering diameter given by Eq. (6) is a computational convenience, and it is not representa-

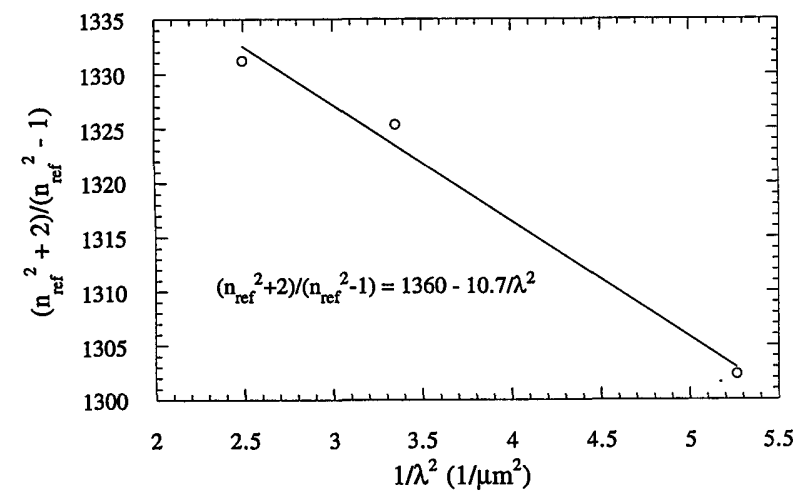

Fig. 2. Measured refractive indices of Freon-12 at $T_{\text {ref }}=0{ }^{\circ} \mathrm{C}$ and $P_{\text {ref }}=1$ atm. tive of any physical dimension of a Freon-12 molecule:

$$
\mathrm{d}_{F}=\left[\frac{9(n+1)^{2}}{\pi^{2} N_{c}^{2}} \frac{6+3 \rho}{6-7 \rho}\right]^{116}
$$

The refractive index and effective scattering diameter were calculated at $1 \mathrm{~atm}$ and $25^{\circ} \mathrm{C}$ for $\lambda=0.84$ $\mu \mathrm{m}$. For these conditions the refractive index is 1.001023 , and the effective scattering diameter is $4.23 \mathrm{~nm}$. We determine the average differential scattering cross section for each detector by considering particles at a large number of positions within the scattering volume. An analysis of the nephelometer geometry gives the scattering angles and solid angles subtended by the detectors at each of these positions. ${ }^{1}$ Differential scattering cross sections are calculated through the use of Mie theory, and they are averaged over the solid angle for each position. We then calculate the average differential scattering cross sections by averaging the values obtained at each particle position. We doubled the number of particle positions until the average differential scattering cross sections converged to within $1 \%$ to prove grid independence.

Theoretical angular scattering cross sections can now be calculated by the use of Eq. (4). The $C_{j}$ are directly proportional to the ratio of the measured scattered power to the incident laser power. We obtain the ratio of the scattered power to the incident laser power by subtracting the stray light SL from each measurement, dividing by the beam dump voltage $\mathrm{BD}$, and averaging over the different laser power settings:

$$
M_{j}^{c}=\frac{1}{p} \sum_{i=1}^{p} \frac{M_{j i}-\mathrm{SL}_{j i}}{\mathrm{BD}_{i}} .
$$

We can use corrected measurements of the light scattered by Freon-12 to calculate absolute scattering correction factors SCF's for each detector. Eq. (8) defines the scattering correction factors:

$$
C_{j}=\mathrm{SCF}_{j} M_{j}^{c} .
$$

Figure 3 is a schematic of the experimental setup for the calibration measurements. We use the nephelometer pump unit to send filtered air back through the nephelometer and out the inlet tube. Once the nephelometer is thoroughly purged, the stray light received by each detector is measured at several laser power levels. Freon-12 from an air conditioner recharging kit is then used to fill the nephelometer as shown in Fig. 3. The Freon-12 was sent through a long coil of tubing before being introduced into the nephelometer so the gas could reach room temperature. We measured the temperature of the gas with a thermocouple just before it entered the nephelometer to ensure that it was at room temperature. The gas was also sent through a $0.2-\mu \mathrm{m}$ filter so that any impurities were removed. The flow of the Freon-12 was regulated such that a slight amount of gas was spilling out of the nephelometer's inlet tube. Re- 


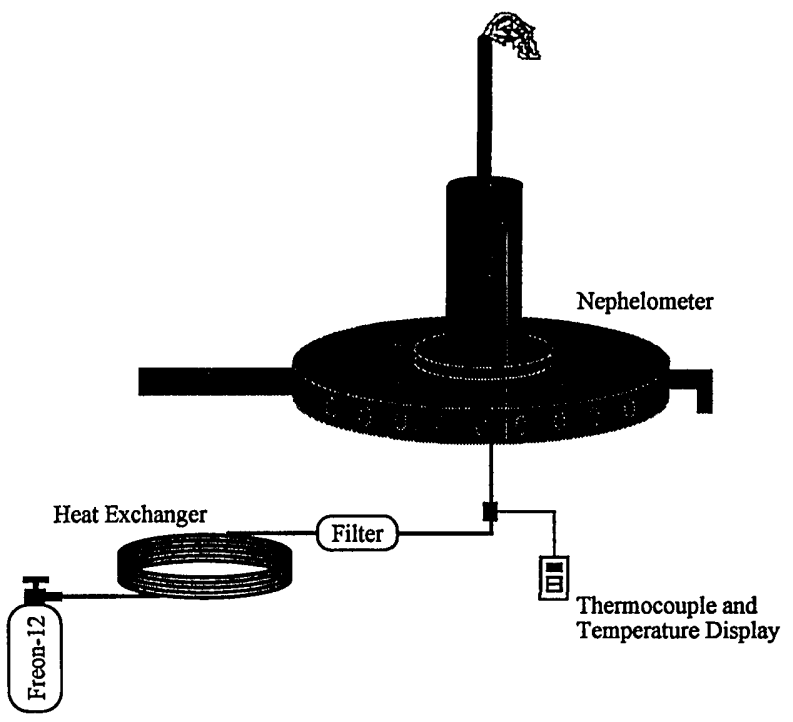

Fig. 3. Schematic diagram for calibration measurements.

cently legislation has been enacted that makes it illegal for chlorofluorocarbons to be vented, so future use of this calibration technique will require that the Freon-12 be recaptured. The light scattered by the Freon-12 was then measured at the same laser power levels as the stray-light measurements.

The uncertainty in the scattering correction factors is determined by the uncertainty in the calculation of the theoretical angular scattering correction factors and the uncertainty in the light-scattering measurements used in the calibration process as shown in Eq. (9). When calculating the uncertainties in this manner, we assume that all the possible errors make positive contributions to the total uncertainty. Therefore, these are conservative estimates of the uncertainties, and the actual errors will be somewhat less than the calculated uncertainties. A detailed discussion of the calculation of each term in Eq. (9) is given by Jones. ${ }^{1}$

$$
\frac{\delta C_{j}}{C_{j}}=\left[\frac{\delta N_{c}}{N_{c}}+\frac{\delta V_{j}}{V_{j}}+\frac{\delta\left(\Delta \Omega_{j}\right)}{\Delta \Omega_{j}}+\frac{\delta\left(\frac{\mathrm{d} \tilde{C}_{j}^{\text {avg }}}{\mathrm{d} \Omega}\right)}{\frac{\mathrm{d} \tilde{C}_{j}^{\text {avg }}}{\mathrm{d} \Omega}}\right] .
$$

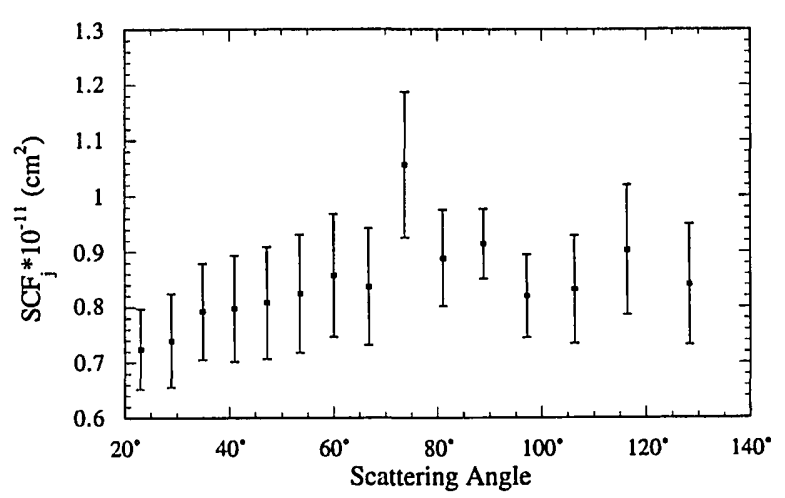

Fig. 4. 'Typical scattering correction factors.

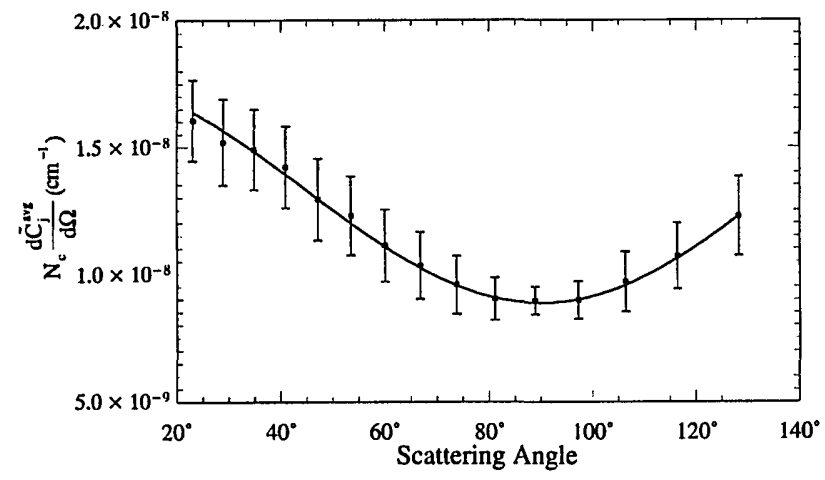

Fig. 5. Light-scattering pattern for dust-off.

We calculate the relative uncertainty in the scattering correction factors by using the uncertainty in the theoretical angular scattering cross sections and the uncertainty in the measurements of the light scattered by Freon-12:

$$
\frac{\delta \mathrm{SCF}_{j}}{\mathrm{SCF}_{j}}=\frac{\delta C_{j}}{C_{j}}+\frac{\delta M_{j}^{c}}{M_{j}^{c}} .
$$

Figure 4 shows a set of typical scattering correction factors. The scattering correction factors depend on various nephelometer settings such as the laser alignment, so these factors will change slightly each time the nephelometer is calibrated.

\section{Aerosol Light-Scattering Measurements}

As a check on the calibration procedure, we obtained absolute measurements of the light scattered by different media and compared them with expected results. The measured angular scattering cross sections were converted into the product of the particle concentration and the average differential scattering cross sections as a way to aid these comparisons.

$$
N_{c} \frac{\mathrm{d} \tilde{C}_{j}^{\text {avg }}}{\mathrm{d} \Omega}=\frac{C_{j}}{\Delta \Omega_{j} V_{j}}
$$

Figure 5 shows the measurements of the light scattered by Dust-Off, a mixture of chlorodifluoromethane and dimethyl ether that is used in cleaning optics. The measured scattering pattern is proportional to $1+\cos ^{2} \theta$, as expected. Figure 6 shows the

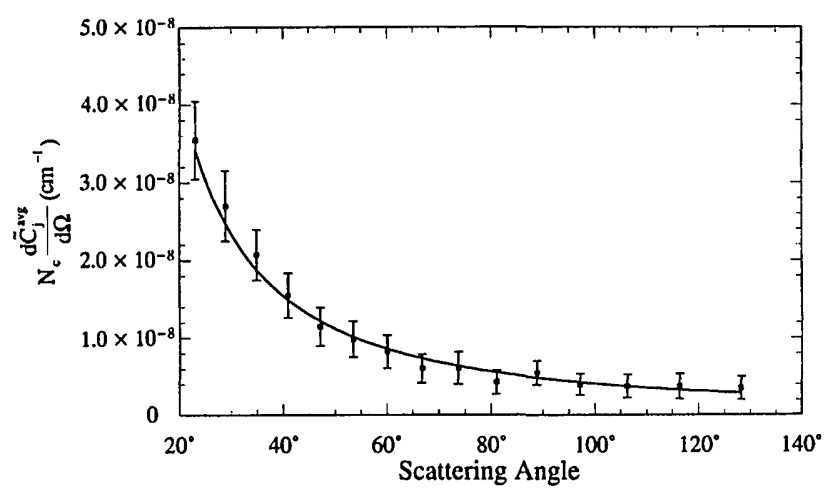

Fig. 6. Light-scattering pattern for room air. 


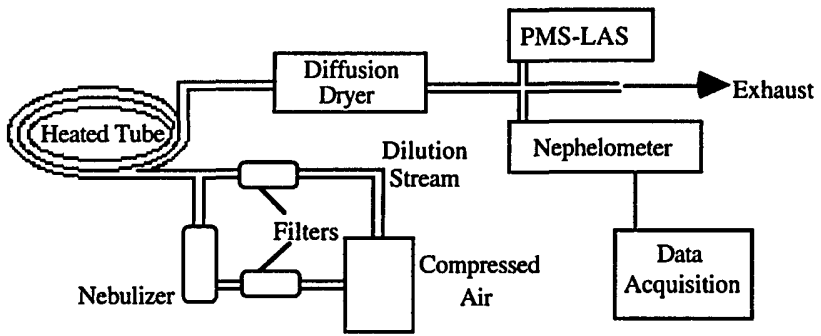

Fig. 7. Schematic diagram for polystyrene sphere light-scattering measurements; PMS-LAS, optical particle counter, Particle Measuring Systems Model LAS-X CRT.

measurements of the light scattered by aerosols suspended in room air. As expected, the scattering pattern is smooth because of the broad size distribution and peaks in the forward-scattering angles.

\section{Polystyrene Sphere Light-Scattering Measurements}

The measurement of the light scattered from polystyrene spheres with known sizes and optical properties will further demonstrate that the nephelometer is properly calibrated. Inversion of these measurements will demonstrate the accuracy of the inversion technique developed in the theoretical portion of this study. Figure 7 is a schematic diagram of the experimental setup for the polystyrene sphere lightscattering measurements.

The polystyrene spheres were diluted with distilled, filtered water and nebulized with a medical nebulizer. The aerosol stream produced by the nebulizer was diluted with filtered compressed air. The water evaporated as the aerosol stream passed through a coil of heated copper tubing, and the moisture was then removed from the stream with a diffusion dryer. After passing through the dyer, the samples from the aerosol stream were drawn into the nephelometer and an optical particle counter (Particle Measuring Systems, Model LAS-X CRT). To ensure that all the moisture was removed from the aerosol stream and that the sediments contained in the water would not significantly affect the light-scattering measurements, we nebulized and analyzed clean water with the nephelometer and the optical particle counter.

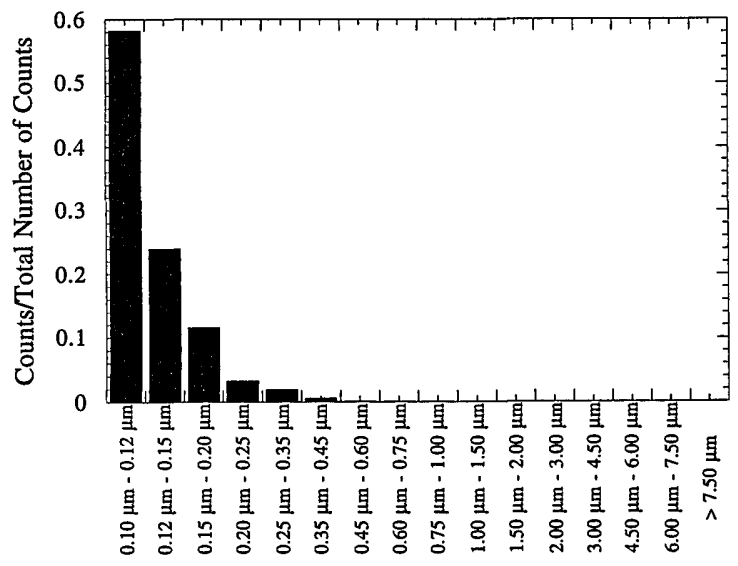

Fig. 8. Histogram for background particles.

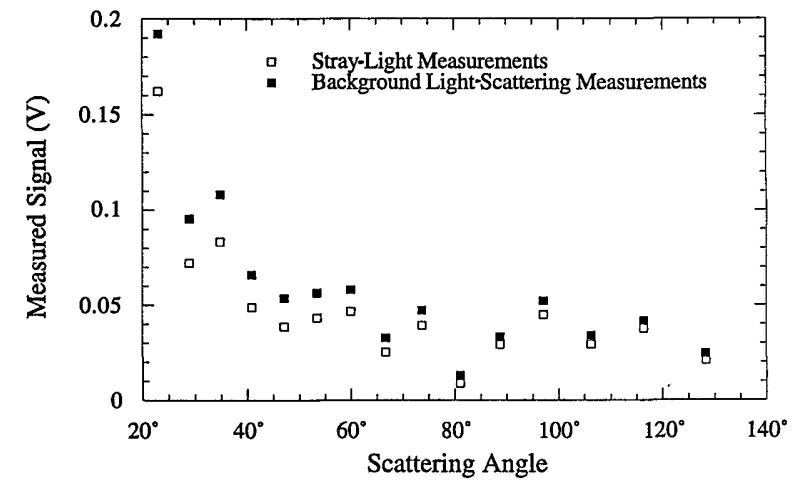

Fig. 9. Comparison of background scattering and stray-light measurements.

The histogram shown in Fig. 8 indicates that there were still some very small $(\leq 0.45 \mu \mathrm{m})$ water droplets or sediments remaining in the aerosol stream. The light scattered by these background particles was monitored for $5 \mathrm{~min}$ and found to be steady. Comparison of these measurements with stray-light measurements (Fig. 9) shows that the difference between the background and the stray light is at most $0.03 \mathrm{~V}$. Because the average measured signal in these experiments is approximately $2 \mathrm{~V}$, the scattering caused by the extraneous particles does not significantly affect the light-scattering measurements. The background scattering measurements were constant with time and were used in place of the stray-light measurements when we reduced the data [see Eq. (7)].

Light-scattering measurements of polystyrene spheres with nominal diameters of 1.06 and $2.02 \mu \mathrm{m}$ were obtained and inverted. Figure 10 shows the measured scattering pattern for the $1.06-\mu \mathrm{m}$ spheres, and Fig. 11 shows the measured scattering pattern for the 2.02- $\mu \mathrm{m}$ spheres. The retrieved size distributions are compared with particle size histograms obtained with the optical particle counter. Figure 12(a) shows the retrieved PSDF for the $1.06-\mu \mathrm{m}$ spheres, and Fig. 12(b) is the corresponding histogram. Figures 13(a) and 13(b) are the retrieved PSDF and histogram for the 2.02- $\mu \mathrm{m}$ spheres, respectively.

As shown in Table 1, the retrieved refractive index compares well with published values for polystyrene spheres. ${ }^{7}$ The retrieved value of the absorption in-

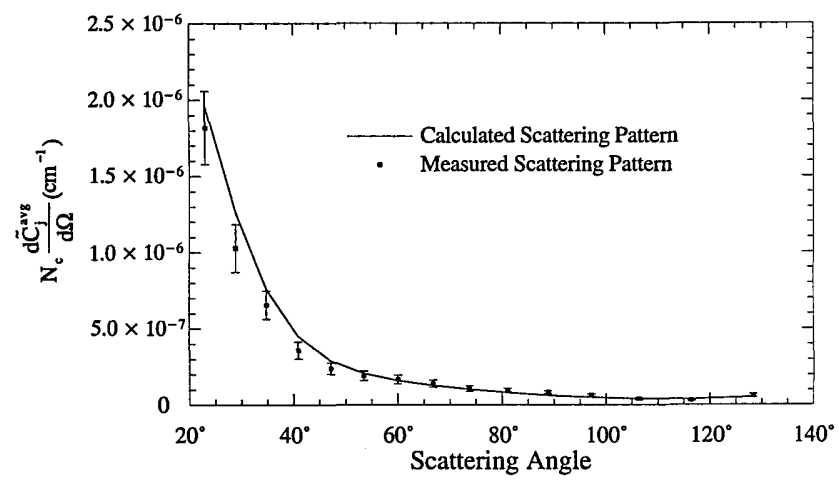

Fig. 10. Light-scattering pattern for $1.06-\mu \mathrm{m}$ polystyrene spheres. 


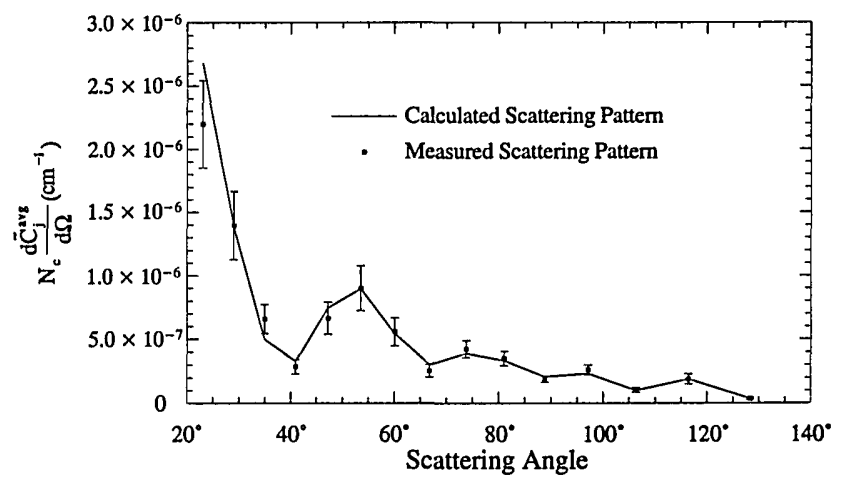

Fig. 11. Light-scattering pattern for $2.02-\mu \mathrm{m}$ polystyrene spheres.

dex is much larger than published values. ${ }^{8}$ Because smaller values of the absorption index give essentially the same rms errors in the calculated scattering patterns, the retrieved values for the absorption index represent upper bounds. These results indicate that light-scattering measurements alone are not sufficiently sensitive to variations in the absorption index to permit the accurate retrieval of this parameter. Incorporation of an absorption or total extinction measurement into the inversion procedure may provide sufficient information to permit the accurate retrieval of the absorption index.

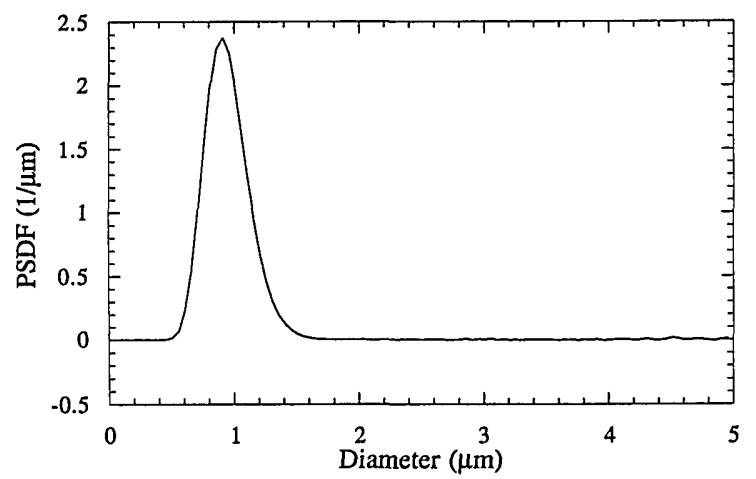

(a)

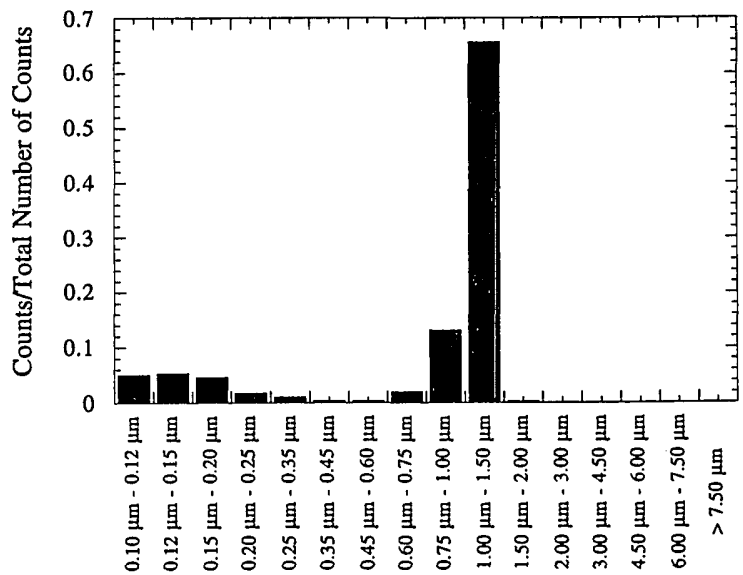

(b)

Fig. 12. 1.06- $\mu \mathrm{m}$ polystyrene spheres: (a) retrieved PSDF, (b) histogram.

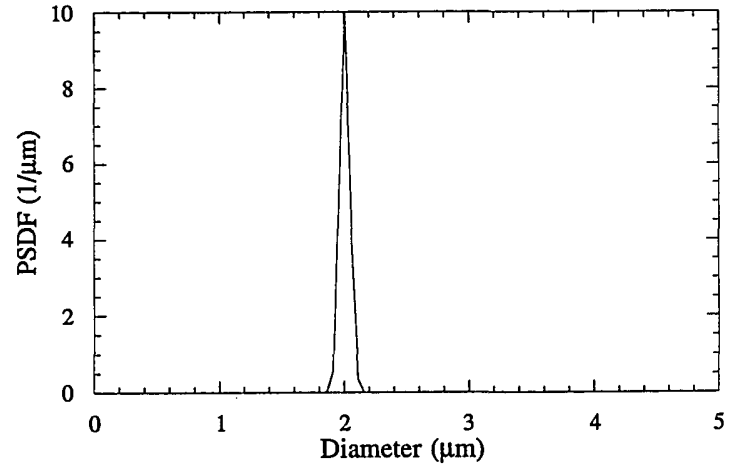

(a)

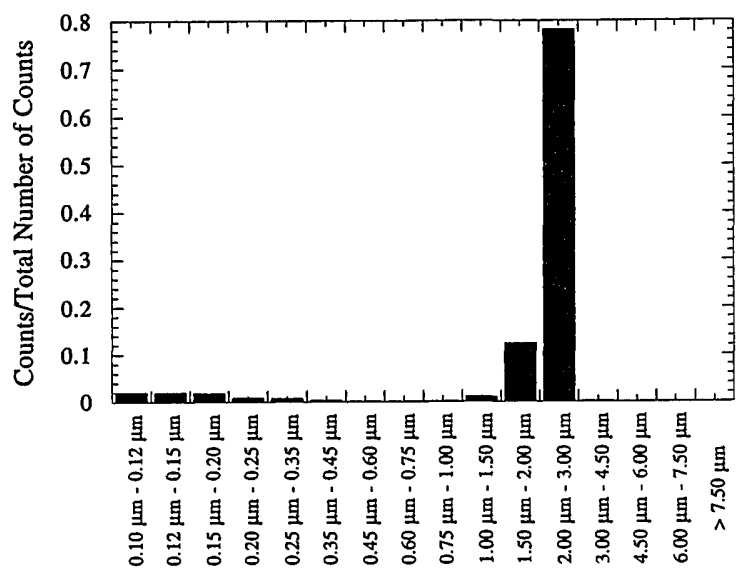

(b)

Fig. 13. 2.02- $\mu \mathrm{m}$ polystyrene spheres: (a) retrieved PSDF, (b) histogram.

The scattering pattern based on the retrieved PSDF and optical properties was calculated with the Mie code written by Bohren and Huffman. ${ }^{3}$ Figure 10 compares the measured scattering pattern to the calculated scattering pattern for the $1.06-\mu \mathrm{m}$ polystyrene spheres. The measured and calculated scattering patterns for the $2.02-\mu \mathrm{m}$ polystyrene spheres are compared in Fig. 11. Figures 10 and 11 show that the measured and calculated scattering patterns agree very well for scattering angles greater than $50^{\circ}$. The scattering pattern based on the retrieved PSDF and optical properties tends to be slightly higher than the measured scattering pattern for scattering angles less than $50^{\circ}$. Because the forward-scattering angles are more sensitive to variations in the size of the particles, the discrepancy at the forward angles was probably caused by the assumption that the PSDF was a log normal distribution. ${ }^{1}$

Table 1. Optical Properties of Polystyrene Spheres

\begin{tabular}{cccc}
\hline & & \multicolumn{2}{c}{ Retrieved Value } \\
\cline { 3 - 4 }$(\lambda=0.84 \mu \mathrm{m})$ & $\begin{array}{c}\text { Reported } \\
\text { Value }\end{array}$ & $1.06-\mu \mathrm{m}$ Spheres & $2.02-\mu \mathrm{m}$ Spheres \\
\hline Refractive Index & $1.57^{a}$ & 1.56 & 1.59 \\
Absorption Index & $0.0^{b}$ & $<10^{-4}$ & $<10^{-4}$ \\
\hline
\end{tabular}

${ }^{a}$ Ref. 8.

${ }^{b} \operatorname{Ref} .9$. 


\section{Conclusions and Recommendations}

The primary difficulty associated with using lightscattering techniques in the determination of aerosol properties is extracting the desired information from the measured data. This paper presented the experimental portion of a study that resulted in the development of a technique capable of retrieving the PSDF and optical properties of aerosol particles from lightscattering measurements. A technique used in the calibration of a multichannel polar nephelometer for absolute scattering measurements was also discussed. The inversion technique was applied to measurements of the light scattered by two monodispersions of polystyrene spheres. The results of these inversions demonstrate that the inversion scheme developed in this study can successfully retrieve the PSDF and refractive index of monodisperse polystyrene spheres. Attempts to retrieve the absorption index were less successful. Because of the relative insensitivity of light-scattering measurements to variations in the absorption index of weakly absorbing particles, only an upper bound on the absorption index could be determined. Increased sensitivity to the absorption index may be obtained by the incorporation of an absorption measurement or a total extinction measurement into the inversion scheme. Shaw ${ }^{9}$ discussed the combined use of angular scattering and spectral extinction measurements in an inversion technique. The possibility that spectral extinction measurements may provide the additional information needed to retrieve the absorption index accurately should be examined further.

\section{Appendix A: Symbols Used in This Paper}

BD, Laser power measured by the beam dump detector $(\mathrm{V})$

$C$, Angular scattering cross section or the ratio of the scattered power received by a detector to the incident irradiance $\left(\mathrm{cm}^{2}\right)$

$\begin{aligned} \frac{\mathrm{d} C}{\mathrm{~d} \Omega}(\Omega, x, \tilde{n}), & \begin{array}{l}\text { Differential scattering cross-sec- } \\ \text { tion detector }\left(\mathrm{cm}^{2}\right)\end{array} \\ \mathrm{d}_{F}, & \begin{array}{l}\text { Effective diameter for Freon-12 } \\ \text { molecules }(\mu \mathrm{m})\end{array} \\ k, & \text { Absorption index } \\ M, & \text { Raw measurement }(\mathrm{V}) \\ M^{c}, & \text { Corrected measurement } \\ n, & \text { Real part of the refractive index } \\ \tilde{n}, & \text { Complex refractive index =n - ik } \\ N_{c}, & \text { Particle number concentration } \\ p, & \left.\text { (cm }{ }^{-3}\right) \\ \mathrm{SCF}, & \text { Scattering correction factor } \\ \mathrm{SL}, & \text { Stray light (V) }\end{aligned}$

$T, \quad$ Temperature (K)

$V$, Volume $\left(\mathrm{cm}^{3}\right)$

$x$, Size parameter $=\pi \mathrm{d} / \lambda$

Greek

$\phi$, Azimuthal angle (deg)

$\lambda$, Wavelength of the incident light $(\mu \mathrm{m})$

$\theta, \quad$ Polar or scattering angle (deg)

$\rho$, Depolarization factor; for Freon$12, \rho=0.048$

Subscripts

$\Omega, \quad$ Solid angle (sr)

Prefix

$\begin{array}{cl}F, & \text { Freon-12 } \\ i, & \text { Laser power level } \\ j, & \text { Detector number }\end{array}$

$\begin{array}{ll}\delta, & \text { Uncertainty } \\ \Delta, & \text { Incremental }\end{array}$

This research was supported by the U.S. Army Chemical Research, Development, and Engineering Center and by the Defense Advanced Research Projects Agency. M. Jones is grateful for the continued support of the Division of Educational Programs at Argonne National Laboratory. The authors thank D. J. Holdridge for preparing some of the figures.

\section{References}

1. M. R. Jones, "Inversion of light scattering measurements for particle size and optical constants," Ph.D. dissertation (University of Illinois at Urbana-Champaign, Urbana, Ill., 1993).

2. M. R. Jones, B. P. Curry, M. Q. Brewster, and K. H. Leong, "Inversion of light-scattering measurements for particle size and optical constants: theoretical study," Appl. Opt. 33, (1994).

3. C. F. Bohren and D. R. Huffman, Absorption and Scattering of Light by Small Particles (Wiley, New York, 1983), Chap. 4, p. 82.

4. B. A. Bodhaine, "Measurement of the Rayleigh scattering properties of some gases with a nephelometer," Appl. Opt. 18, 121-125 (1979).

5. M. Q. Brewster, Thermal Radiative Transfer \& Properties (Wiley, New York, 1992), Chap. 5, p. 157.

6. R. W. Ditchburn, Light, 2nd ed. (Interscience, New York, 1963, Chap. 15, p. 549.

7. M. J. Devon and A. Rudin, "A simple technique for measuring the refractive index of polymer latexes at various wavelengths," J. Appl. Polym. Sci. 34, 469-476 (1987).

8. T. Inagaki, E. T. Arakawa, R. N. Hamm, and M. W. Williams, "Optical properties of polystyrene from the near-infrared to the $x$-ray region and convergence of optical sum rules," Phys. Rev. B 15, 3243-3253 (1977).

9. G. E. Shaw, "Inversion of optical scattering and spectral extinction measurements to recover aerosol size spectra," Appl. Opt. 18, 988-993 (1979). 